\title{
Thermodynamics of the Harmonic Oscillator: Derivation of the Planck Blackbody Spectrum from Pure Thermodynamics
}

\author{
Timothy H. Boyer \\ Department of Physics, City College of the City \\ University of New York, New York, New York 10031
}

\begin{abstract}
In 1893, Wien applied the first two laws of thermodynamics to blackbody radiation and derived his displacement theorem. Believing that the information from thermodynamics had been exhausted, Planck turned to statistical ideas in 1900 in order to provide a physical understanding for his experimental-data-based interpolation giving the Planck spectrum without zero-point radiation. Here we point out that the third law of thermodynamics (which was developed in the early years of the 20th century) introduces additional thermodynamic information regarding thermal radiation. The Planck spectrum for thermal radiation can be derived from purely thermodynamic ideas applied to the classical simple harmonic oscillator, since every radiation mode takes a simple oscillator form. Thermodynamics alone implies the Planck spectrum including zero-point energy without any need for quantum theory or statistical ideas. It is pointed out that the inclusion of zero-point energy involves more natural thermodynamics than its exclusion.
\end{abstract}




\section{INTRODUCTION}

At the end of the 19th century, there were only two laws of thermodynamics. The first law introduced heat energy into the ideas of energy conservation. The second law involved the existence of an entropy state function and an absolute temperature scale. In 1893, these two laws were used by Wien to derive his displacement theorem for the blackbody radiation spectrum. [1] However, Wien's displacement theorem did not solve the blackbody radiation problem because there still remained an unknown function of a single variable involving frequency divided by absolute temperature. In 1900, when Planck became convinced that the useful information from the laws of thermodynamics had been exhausted, he turned to statistical mechanics to give a physical basis for his interpolation guess for the blackbody

radiation spectrum. [2] Planck's use of statistical ideas led to the beginnings of quantum theory. It is striking that within a decade of Planck's work, Nernst's heat theorem associated with the low-temperature specific heats of solids gave the basis for what is now termed the third law of thermodynamics. [3] Although the textbooks of thermodynamics and statistical mechanics regard the third law as related to quantum theory, [4] the third law also fits into classical theory. [5] Indeed it turns out that the use of all three laws of thermodynamics allows a complete derivation of the Planck spectrum based upon pure thermodynamics without any recourse whatsoever to a statistical theory. The basic thermodynamics and mathematics of the analysis is elementary and can be followed by an advanced undergraduate physics student.

\section{OUTLINE OF THE ANALYSIS}

Our analysis makes use of information about classical harmonic oscillators, the laws of thermodynamics, and applications of analytic function theory. We start by noting that a classical harmonic oscillator in a thermodynamic bath can be regarded as a thermodynamic system with an energy, generalized force, and temperature. The classical oscillator has an adiabatic invariant given by the energy divided by the frequency, and this adiabatic invariant allows us to recognize the generalized force associated with the work done by an adiabatic change in the spring constant (or of the frequency). Thus a classical harmonic oscillator in a heat bath can be treated as a very simple thermodynamic system of two independent 
variables, energy $U$ and frequency $\omega$. Next we turn to the laws of thermodynamics, starting with the existence of an empirical temperature and then using the first two laws to establish that the entropy of the oscillator is a function of a single variable involving the energy divided by the frequency. Next we connect the behavior of the oscillator at large energy with the thermodynamic temperature. The proportionality of the thermodynamic temperature and the oscillator energy at high temperature allows us to obtain the functional form of the oscillator entropy at large energy. We next assume that the entropy is an analytic function of energy and apply the third law of thermodynamics to the analytic form. We find that in order to satisfy the third law of thermodynamics, the oscillator must have finite energy at zero thermodynamic temperature. The behavior of the derivatives of the entropy near zero temperature suggests singular terms in the entropy as a function of energy. Making use of the singularities and comparing with the assumed high-temperature form obtained earlier, we are able to obtain the Planck spectrum with zero-point energy as the simplest entropy expression consistent with the three laws of thermodynamics; any additional terms would involve additional singularities in the complex plane. Finally we comment on the classical thermodynamics which we have obtained in relation to the corresponding quantum expressions. The article closes with brief historical remarks regarding the third law of thermodynamics.

\section{HARMONIC OSCILLATORS AS THERMODYNAMIC SYSTEMS}

Elementary discussions of thermodynamics often refer to gas systems with two independent variables, pressure and volume, whereas the simple harmonic oscillator is usually introduced only in connection with statistical mechanics. [6] Nevertheless, the classical simple harmonic oscillator provides a very simple thermodynamic system of two independent variables, energy $U$ and frequency $\omega$. A mechanical oscillator has an equation of motion $m \ddot{x}=-k x$, an oscillator (angular) frequency $\omega=(k / m)^{1 / 2}$, and an energy $U=(1 / 2) m \dot{x}^{2}+(1 / 2) k x^{2}$. It can be shown that the oscillator energy divided by the frequency, $U / \omega=U(m / k)^{1 / 2}$, is

an adiabatic invariant [7] for the mechanical system, so that under a very slow change of the spring constant $k$, the quantity $U(m / k)^{1 / 2}$ remains unchanged. Therefore we have

$$
0=d U(m / k)^{1 / 2}-d k\left[U\left(m / k^{3}\right)^{1 / 2} / 2\right]=d U / \omega-d \omega\left(U / \omega^{2}\right) .
$$


The expressions involving frequency $\omega$ are simpler in form than those involving the spring constant $k$, and so we will refer to the change in the oscillator frequency $\omega$ rather than to the change in the spring constant $k$. The change in energy $d U$ associated with the change $d \omega$ in the oscillator frequency $\omega$ must be provided by some external agent. Then from Eq. (1), the work $d W$ done by the oscillator is just the negative of this agent's work, $d W=-$ $(U / \omega) d \omega$, and therefore

$$
X=-U / \omega
$$

can be regarded as a generalized force associated with the oscillator.

A classical harmonic oscillator can be regarded as coming to equilibrium in a thermal bath and so providing a simple thermodynamic system. Equation (2), connecting $X, U$, and $\omega$, may be regarded as an equation of state for the system. We will regard thermal radiation as providing the thermal bath, and each oscillator is treated as a small electric dipole oscillator with a very small electric charge interacting with electromagnetic radiation. At the end of the 19th century, Planck showed[8] that a small electric dipole oscillator came to equilibrium with random radiation when the average energy of the oscillator matched the average energy of the radiation-bath normal modes at the same frequency as that of the oscillator.

\section{ZEROTH LAW: EMPIRICAL TEMPERATURE FOR A HARMONIC- OSCILLATOR SYSTEM}

We will consider a collection of oscillators at a various mechanical frequencies $\omega$, where for each oscillator the average energy is given by its energy $U$, and the generalized force $X$ is given by $X=-U / \omega$. The zeroth law of thermodynamics introduces the idea of an empirical temperature $\theta(U, \omega)$ associated with the average oscillator energy $U$ and the mechanical frequency $\omega$. If two oscillators of different frequencies $\omega$ and $\omega^{\prime}$ agree on the empirical temperature, $\theta(U, \omega)=\theta\left(U^{\prime}, \omega^{\prime}\right)$, then the two oscillators will be in thermal equilibrium with the same thermal radiation bath and in thermal equilibrium with each other.

\section{FIRST LAW OF THERMODYNAMICS}

The first law of thermodynamics introduces the idea that heat energy $\delta Q$ can be included in the conservation law for energy, as $\delta Q=d U+\delta W$. For a harmonic oscillator, this 
conservation equation takes the form

$$
\delta Q=d U-(U / \omega) \delta \omega
$$

where $\delta Q$ corresponds to the heat energy added to the oscillator, $U$ is the internal energy of the oscillator, and $-(U / \omega) \delta \omega$ corresponds to the work done by the oscillator. The internal energy $U(\theta, \omega)$ of an oscillator is a function of the empirical temperature $\theta$ and the frequency $\omega$ of the oscillator. We will now define the empirical temperature $\theta$ by choosing an oscillator of a single preferred frequency $\omega_{*}$ and taking the average energy $U_{*}$ of this oscillator as equal to the empirical temperature, $\theta=U_{*}$. For this oscillator, as more heat energy $\delta Q$ is added to the system, the energy $U_{*}$ will increase for fixed $\omega_{*}$ according to Eq. (3) . In this fashion, we assure that the empirical temperature $\theta=U_{*}=\theta\left(U_{*}, \omega_{*}\right)=\theta(U, \omega)$ is an increasing function of energy for the oscillator at the special frequency $\omega_{*}$.

\section{SECOND LAW OF THERMODYNAMICS}

For our harmonic oscillator system, the second law of thermodynamics declares that there exists an entropy state function $S(U, \omega)$ which is a function of the average oscillator energy $U$ and of the oscillator frequency $\omega$, such that

$$
T d S=d U-(U / \omega) d \omega
$$

where

$$
T=(\partial U / \partial S)_{\omega}
$$

is a thermodynamic temperature which depends only upon the empirical temperature $\theta$. Here if we introduce the relation (5) into the second-law relation (4) divided by $T$, then we find

$$
d S=\frac{1}{T} d U-\frac{U}{T \omega} d \omega=\left(\frac{\partial S}{\partial U}\right)_{\omega} d U-\frac{U}{\omega}\left(\frac{\partial S}{\partial U}\right)_{\omega} d \omega
$$

However, if we regard $S$ as a function of $U$ and $\omega$, so that $d S=(\partial S / \partial U)_{\omega} d U+(\partial S / \partial \omega)_{U} d \omega$, then the relation (6) requires

$$
\left(\frac{\partial S}{\partial \omega}\right)_{U}=-\frac{U}{\omega}\left(\frac{\partial S}{\partial U}\right)_{\omega}
$$

which means that $S$ is a function of the single variable $(U / \omega)$. Thus we have

$$
\left(\frac{\partial S(U / \omega)}{\partial \omega}\right)_{U}=-\frac{U}{\omega^{2}} S^{\prime}(U / \omega)=-\frac{U}{\omega}\left(\frac{\partial S(U / \omega)}{\partial U}\right)_{\omega} .
$$

where $S^{\prime}(U / \omega)$ refers to the derivative of $S(U / \omega)$ with respect to its one argument $U / \omega$. 


\section{ADIABATIC LINES}

A reversible adiabatic process involving a harmonic oscillator involves work being done without the addition of heat. Under such a process, the entropy of the system remains unchanged since $\delta Q=T d S=0$. Since for a harmonic-oscillator thermodynamic system, the entropy $S$ is a function of $U / \omega$, the adiabatic lines will be straight lines through the origin in a plot of average energy $U$ vs $\omega$, or will be horizontal straight lines in an indicator plot of the generalized force $U / \omega=-X$ vs $\omega$.

\section{CONNECTING EMPIRICAL AND THERMODYNAMIC TEMPERATURE}

In order to connect the empirical temperature $\theta$ with thermodynamic temperature $T$, we can follow the basic form of analysis given in Sears and Salinger for a gas system. [9] If we regard the average energy $U$ of an oscillator as a function of frequency and thermodynamic temperature, then we have $d U=(\partial U / \partial T)_{\omega} d T+(\partial U / \partial \omega)_{T} d \omega$. Substituting this expression into the second-law equation (44), we obtain

$$
d S=\frac{1}{T}\left(\frac{\partial U}{\partial T}\right)_{\omega} d T+\frac{1}{T}\left[\left(\frac{\partial U}{\partial \omega}\right)_{T}-\frac{U}{\omega}\right] d \omega .
$$

Now comparing this expression with $d S=(\partial S / \partial T)_{\omega} d T+(\partial S / \partial \omega)_{T} d \omega$, gives

$$
\left(\frac{\partial S}{\partial T}\right)_{\omega}=\frac{1}{T}\left(\frac{\partial U}{\partial T}\right)_{\omega} \text { and }\left(\frac{\partial S}{\partial \omega}\right)_{T}=\frac{1}{T}\left[\left(\frac{\partial U}{\partial \omega}\right)_{T}-\frac{U}{\omega}\right] .
$$

Using the agreement of the mixed second-partial derivatives of $S$ with respect to $T$ and $\omega$ in opposite orders, we have

$$
\left\{\frac{\partial}{\partial \omega}\left[\frac{1}{T}\left(\frac{\partial U}{\partial T}\right)_{\omega}\right]\right\}_{T}=\left\{\frac{\partial}{\partial T}\left[\frac{1}{T}\left\{\left(\frac{\partial U}{\partial \omega}\right)_{T}-\frac{U}{\omega}\right\}\right]\right\}_{\omega} .
$$

Next simplifying this expression and canceling the mixed second derivatives of $U$ on the two sides, we have[11]

$$
\left(\frac{\partial U}{\partial \omega}\right)_{T}=-\frac{T}{\omega}\left(\frac{\partial U}{\partial T}\right)_{\omega}+\frac{U}{\omega}
$$

or equivalently

$$
\frac{U}{\omega}=f(T / \omega)
$$

where $f(T / \omega)$ is some function of the single variable $T / \omega$. The information in Eq. (13)

corresponds to that of Wien's displacement theorem. [10] 
We now use equation (12) to connect the empirical and thermodynamic temperatures for the harmonic oscillator. The thermodynamic temperature $T$ is some function of the empirical temperature $\theta$ alone, so that $(\partial \theta / \partial T)_{\omega}=d \theta / d T$. Thus our equation (12) becomes

$$
\left(\frac{\partial U}{\partial \omega}\right)_{\theta}=-\frac{T}{\omega}\left(\frac{\partial U}{\partial \theta}\right)_{\omega} \frac{d \theta}{d T}+\frac{U}{\omega} .
$$

This implies

$$
\frac{d T}{T}=\frac{1 / \omega(\partial U / \partial \theta)_{\omega}}{U / \omega-(\partial U / \partial \omega)_{\theta}} d \theta=\frac{(\partial U / \partial \theta)_{\omega}}{U-\omega(\partial U / \partial \omega)_{\theta}} d \theta .
$$

The left-hand side of this equation is a function of $T$ only, and therefore the right-hand side must be a function of $\theta$ only and must be independent of $\omega$. Therefore we may choose to evaluate each term of the right-hand side at $\omega=\omega_{*}$ where $\omega_{*}$ refers to the special oscillator whose energy $U_{*}$ is used as the empirical temperature $\theta$. Thus when $\omega=\omega_{*}$, we have $\theta=U_{*}$, $\left(\partial U_{*} / \partial \theta\right)_{\omega_{*}}=1$, and $d \theta=d U_{*}$. The equation (15) then becomes

$$
\frac{d T}{T}=\frac{1}{U_{*}-\omega_{*}\left(\partial U_{*} / \partial \omega_{*}\right)_{\theta}} d U_{*} .
$$

Eventually for large enough energies $U_{*}$, we expect $U_{*}$ to become much larger than the magnitude of $\omega_{*}\left(\partial U_{*} / \partial \omega_{*}\right)_{\theta}$ unless the slope of isothermals at $\omega_{*}$ increases steadily with energy $U_{*}$, which seems completely unphysical. Indeed, we expect that at high temperature, the quantity $\left(\partial U_{*} / \partial \omega_{*}\right)$ should become ever smaller

$$
\left(\frac{\partial U_{*}}{\partial \omega_{*}}\right)_{\theta} \approx O\left(\frac{\omega_{*}}{U_{*}}\right) \text { for large } U_{*},
$$

corresponding to independence of the oscillator energy from the oscillator frequency. But then we have

$$
\begin{aligned}
\frac{d T}{T} & =\frac{1}{U_{*}\left[1-\left(\omega_{*} / U_{*}\right)\left(\partial U_{*} / \partial \omega_{*}\right)_{\theta}\right]} d U_{*} \\
& =\left\{\frac{1}{U_{*}}+O\left(\frac{\omega_{*}^{2}}{U_{*}^{3}}\right)\right\} d U_{*} .
\end{aligned}
$$

Integrating gives

$$
\ln T=\ln U_{*}+\ln C+O\left(\frac{\omega_{*}^{2}}{U_{*}^{2}}\right)
$$

and

$$
T=C U_{*}+O\left(\frac{\omega_{*}^{2}}{U_{*}^{2}}\right)
$$

where $C$ is a constant. The scale constant $C$ connects thermodynamic temperature $T$ and empirical temperature $\theta=U_{*}$ used above. The usual choice corresponding to "practical 
units" is $C=1 / k_{B}$ where $k_{B}$ is Boltzmann's constant. However, in most of the analysis to follow, it is more convenient to choose $C=1$, corresponding to "rational units," where absolute temperature $T$ is measured in energy units and entropy $S$ is a dimensionless pure number.[12] Since the choice of the special frequency $\omega_{*}$ was arbitrary, we expect this relationship to hold for large values of the oscillator energy, no matter what the frequency of the oscillator

$$
T \rightarrow U\left[1+O\left(\frac{\omega^{2}}{U^{2}}\right)\right] \text { for large } U
$$

Thus at high temperatures, the energy of a harmonic oscillator becomes independent of the frequency $\omega$ of the oscillator. This high-temperature result agrees with the equipartition result found from nonrelativistic classical statistical mechanics.

The entropy expression at all temperatures following from our Eq. (21) follows from

$$
\left(\frac{\partial S}{\partial(U / \omega)}\right)_{\omega}=\frac{\omega}{T}=\frac{\omega}{U\left[1+O\left(\omega^{2} / U^{2}\right)\right]}=\frac{\omega}{U}\left[1+O\left(\frac{\omega^{2}}{U^{2}}\right)\right] .
$$

Integrating once, we have

$$
S(U / \omega)=\ln (U / \omega)+\text { const }+O\left(\frac{\omega^{2}}{U^{2}}\right) .
$$

\section{HIGH-TEMPERATURE ENTROPY-ENERGY CONNECTION ASSUMED AT ALL TEMPERATURES}

The high-temperature limit $U \approx T$ in Eq. (21) between oscillator energy $U$ and thermodynamic temperature $T$ is actually consistent with the first two laws of thermodynamics if the relation $T=U$ is assumed to hold for all temperatures. Thus if we set

$$
T=U \quad \text { assumed for all } T
$$

then we have $(\partial U / \partial \omega)_{\theta}=0$ in Eq. (16) and our analysis in Eqs. (18)-(21) goes through with no correction term. We solve for the entropy function $S(U / \omega)$ using

$$
\frac{1}{T}=\left(\frac{\partial S}{\partial U}\right)_{\omega}
$$

which from Eq. (24) becomes

$$
\frac{\omega}{T}=\frac{\omega}{U}=\left(\frac{\partial S}{\partial(U / \omega)}\right)_{\omega}
$$


or

$$
S(U / \omega)=\ln (U / \omega)+\text { const }
$$

Thus at large values of energy, we expect the entropy to take the form in Eq. (27), the first derivative to give

$$
0<\frac{1}{T}=\left(\frac{\partial S}{\partial U}\right)_{\omega}=\frac{1}{U}
$$

and the second derivative to give

$$
0>\left(\frac{\partial^{2} S}{\partial U^{2}}\right)_{\omega}=\frac{-1}{U^{2}} .
$$

The function (27) indeed satisfies the criteria of the first two laws of thermodynamics.

\section{THIRD LAW OF THERMODYNAMICS AND LOW TEMPERATURES}

Although the first two laws of thermodynamics hold generally at any temperature, the third law of thermodynamics deals specifically with thermodynamic behavior at low temperatures. In particular, there is no assurance that the limiting expression $S(U / \omega)=$ $\ln (U / \omega)+$ const. in (27), which we have obtained for the entropy at high temperatures, will continue to hold as the thermodynamic temperature tends toward absolute zero. Indeed, the third law states that as $(\partial U / \partial S)_{\omega}=T \rightarrow 0$, the values of the entropy fall toward zero, $S(U / \omega) \rightarrow 0$. It is clear that the entropy relation in Eq. (27) does not satisfy the third law of thermodynamics. Thus equation (27) leads to Eq. (28) and so gives $T=U$; yet taking $U=T \rightarrow 0_{+}$in equation (27) gives entropy which diverges to negative infinity, $S=\ln (U / \omega)+$ const $\rightarrow-\infty$ as $U \rightarrow 0$ for fixed $\omega$. Therefore in order to satisfy the third law of thermodynamics, the entropy $S(U / \omega)$ of a harmonic oscillator must depart from the high-temperature form in Eq. (27). We wish to obtain the necessary modifications to the entropy function.

\section{ZERO-POINT ENERGY}

The entropy function given by $S(U / \omega)=\ln (U / \omega)+$ const in Eq. (27) is analytic on the positive real axis in $U / \omega$, and the corresponding temperature (involving derivatives of the 
entropy)

$$
\frac{\omega}{T}=\frac{d S}{d(U / \omega)}=\frac{\omega}{U}
$$

is positive on the entire positive real axis for $U / \omega$; however, the entropy function itself is not positive on the full real axis since $\ln (U / \omega) \rightarrow-\infty$ as $U / \omega \rightarrow 0$. Now we expect the entropy $S(U / \omega)$ to be an analytic function of $U / \omega$, so that working from the high-temperature form, we expect the additional terms to be given by a series expansion in inverse powers of $U / \omega$ so that

$$
S(U / \omega)=\ln (U / \omega)+\text { const }+\sum_{n=1}^{\infty} \frac{a_{n}}{(U / \omega)^{n+1}}
$$

where the $a_{n}$ are constants and the sum involves powers to the $n+1$ because of the correction in Eq. (23) is $O\left(\omega^{2} / U^{2}\right)$. As $U / \omega$ becomes large, each of the terms $(U / \omega)^{-n-1}$ in the sum goes to zero faster than $\ln (U / \omega)$ so that $\ln (U / \omega)$ indeed can dominate at large values of $U / \omega$. However, as $U / \omega$ decreases toward zero, the terms $(U / \omega)^{-n-1}$ in the sum will diverge faster than $\ln (U / \omega)$ and so will dominate the expression. Accordingly, the function $S(U / \omega)$, which must be monotonically increasing with increasing $U / \omega$, cannot be positive and analytic on the entire positive real axis of $U / \omega$. Instead, there must be some positive value of $U / \omega$ where $S(U / \omega)$ becomes zero. Thus there must be a smallest positive value of $U / \omega$ where the thermodynamic temperature $T$ falls to zero. We will take this smallest value as $U / \omega=\hbar / 2$. Thus every oscillator of frequency $\omega$ has a smallest positive energy (a zero-point energy) given by $U_{0}=U(\omega, 0)=\omega \hbar / 2$. Crucially, the third law of thermodynamics, when applied to the harmonic oscillator system, immediately implies the idea of zero-point energy at the absolute zero of temperature. Zero-point energy for a harmonic oscillator is implicit in the third law of thermodynamics.

\section{FORM OF THE ENTROPY FUNCTION}

The third law of thermodynamics requires that the entropy $S$ approaches zero as the temperature $T$ falls toward zero corresponding to $U / U_{0}$ approaching $1_{+}$. We will set $z=$ $U / U_{0}=U /(\omega \hbar / 2)$ so that the entropy in Eq. (31) can be regarded as a function of $z$ with

$$
S(z)=\ln (z)+\text { Const }+\sum_{n=1}^{\infty} \frac{A_{n}}{z^{n+1}},
$$

where Const and the coefficients $A_{n}$ are adjusted from const and the $a_{n}$ in Eq. (31) so as to absorb the constant $\hbar / 2$ associated with the oscillator's zero-point energy. Then it follows 
from Eq. (32) that

$$
0<\frac{\omega \hbar / 2}{T}=\frac{d S}{d z}=\frac{1}{z}+\sum_{n=1}^{\infty} \frac{-(n+1) A_{n}}{z^{n+2}}
$$

and

$$
0>\frac{d^{2} S}{d z^{2}}=-\frac{1}{z^{2}}+\sum_{n=1}^{\infty} \frac{(n+1)(n+2) A_{n}}{z^{n+3}},
$$

where $S(z) \rightarrow 0$ and $T(U, \omega) \rightarrow 0$ as $z \rightarrow 1_{+}$. It follows from Eq.(33) that the entropy function must have a divergent derivative $d S / d z$ as $z \rightarrow 1$ since $(\omega \hbar / 2) / T \rightarrow \infty$ as $T \rightarrow 0$ for fixed $\omega$. Also, if the first derivative of $S(z)$ has a singularity at $z=1$, then the second derivative must have an even stronger singularity. The weakest singularity would be a logarithmic singularity, such as $d S / d z \sim \ln (z-1)$, but the next derivative would give a firstorder singularity as $d^{2} S / d z^{2} \sim 1 /(z-1)$. And indeed for these singularities, the function $S$ itself could have a finite limit at the singularity corresponding to $S \sim(z-1) \ln (z-1) \rightarrow 0$ for $z \rightarrow 1_{+}$.

The known high-temperature behavior places further limits on the low-temperature singular behavior. If we try a first-order singularity at $z=1$ for the second derivative of $S(z)$, we must include an additional first-order singularity at some other value $z=\alpha$ in order that the second derivative of $S$ falls off at large $z$ as the second power of $z$ as in Eq. (34). Thus we can write

$$
\begin{aligned}
\frac{d^{2} S}{d z^{2}} & =-\frac{1}{(z-1)(z-\alpha)}+\sum_{n=1}^{\infty} \frac{(n+1)(n+2) b_{n}}{z^{n+3}} \\
& =\frac{-1}{1-\alpha}\left(\frac{1}{z-1}-\frac{1}{z-\alpha}\right)+\sum_{n=1}^{\infty} \frac{(n+1)(n+2) b_{n}}{z^{n+3}}
\end{aligned}
$$

where the $b_{n}$ are constants, and so preserve the analytic form and high-temperature limit in Eq. (34), provided that the value of $\alpha$ lies on the real axis with $\alpha<1$, which is out of the physical region $1<z$, corresponding to oscillator energies above the zero-point energy.

Integrating once in Eq. (35), we have

$$
\begin{aligned}
\frac{d S}{d z} & =\frac{-1}{1-\alpha}[\ln (z-1)-\ln (z-\alpha)]+\gamma-\sum_{n=1}^{\infty} \frac{(n+1) b_{n}}{z^{n+2}} \\
& =\frac{-1}{1-\alpha}\left[\ln z+\ln \left(1-\frac{1}{z}\right)-\ln z-\ln \left(1-\frac{\alpha}{z}\right)\right]+\gamma-\sum_{n=1}^{\infty} \frac{(n+1) b_{n}}{z^{n+2}} \\
& =\frac{-1}{1-\alpha}\left[-\frac{1}{z}+\frac{1}{2 z^{2}}-\ldots+\frac{\alpha}{z}-\frac{\alpha^{2}}{2 z^{2}}+\ldots\right]+\gamma-\sum_{n=1}^{\infty} \frac{(n+1) b_{n}}{z^{n+2}}
\end{aligned}
$$


where $\gamma$ and the $b_{n}$ are constants. In order for this expression (36) to go over to the hightemperature form given in Eq. (33), we must have $\alpha=-1$ and $\gamma=0$. Introducing these values into the first line in Eq. (36), we obtain

$$
\frac{d S}{d z}=\frac{-1}{2}[\ln (z-1)-\ln (z+1)]-\sum_{n=1}^{\infty} \frac{(n+1) b_{n}}{z^{n+2}} .
$$

Now integrating equation (37) gives

$$
\begin{aligned}
S(z) & =\frac{-1}{2}[(z-1) \ln (z-1)-(z+1) \ln (z+1)]+\text { const }^{\prime}+\sum_{n=1}^{\infty} \frac{b_{n}}{z^{n+1}} \\
& =\frac{-1}{2}\left\{(z-1) \ln z+(z-1) \ln \left(1-\frac{1}{z}\right)-(z+1) \ln z-(z+1) \ln \left(1+\frac{1}{z}\right)\right\} \\
& + \text { const }^{\prime}+\sum_{n=1}^{\infty} \frac{b_{n}}{z^{n+1}} \\
& =\ln z+\frac{-1}{2}\left\{(z-1)\left(\frac{-1}{z}+\frac{1}{2 z^{2}}-\ldots\right)-(z+1)\left(\frac{1}{z}-\frac{1}{2 z^{2}}+\ldots\right)\right\}+\text { const }^{\prime}+\sum_{n=1}^{\infty} \frac{b_{n}}{z^{n+1}} \\
& =\ln z+\text { const }^{\prime \prime}+\sum_{n=1}^{\infty} \frac{c_{n}}{z^{n+1}}
\end{aligned}
$$

where const', const", and $c_{n}$ are all constants. The final line in Eq. (38) has a large- $z$ analytic behavior consistent with that in Eq. (32).

In our analysis starting with the third law of thermodynamics, we see the need for certain singular terms to appear in the derivatives of $S(z)$, and we can then use integrals to trace back the implications for their appearance in the function $S(z)$ itself. If we take only the terms required by the singularities at low temperature while still maintaining the hightemperature limit, then we have the expression for the entropy

$$
S(z)=\frac{-1}{2}[(z-1) \ln (z-1)-(z+1) \ln (z+1)]-\ln 2
$$

which vanishes as $z$ approaches $1, z \rightarrow 1_{+}$. This function (39) can be rewritten in the high-temperature form given in Eq. (32) as 


$$
\begin{aligned}
S(z) & =\frac{-1}{2}[(z-1) \ln (z-1)-(z+1) \ln (z+1)]-\ln 2 \\
& =\frac{-1}{2}\left\{(z-1) \ln z+(z-1) \ln \left(1-\frac{1}{z}\right)-(z+1) \ln z-(z+1) \ln \left(1+\frac{1}{z}\right)\right\}-\ln 2 \\
& =\ln z+\frac{-1}{2}\left\{(z-1)\left(\frac{-1}{z}+\frac{1}{2 z^{2}}-\ldots\right)-(z+1)\left(\frac{1}{z}-\frac{1}{2 z^{2}}+\ldots\right)\right\}-\ln 2 \\
& =\ln z+1-\ln 2-\frac{1}{6 z^{2}}-\frac{1}{20 z^{4}}-\frac{1}{42 z^{6}}-\ldots \text { for } 1<z .
\end{aligned}
$$

The derivatives follow as

$$
\begin{aligned}
\frac{d S}{d z} & =\frac{-1}{2}[\ln (z-1)-\ln (z+1)]=\frac{1}{2} \ln \left(\frac{z+1}{z-1}\right) \\
& =\frac{1}{z}+\frac{1}{3 z^{3}}+\frac{1}{5 z^{5}}+\ldots>0 \text { for } 1<z
\end{aligned}
$$

and

$$
\begin{aligned}
\frac{d^{2} S}{d z^{2}} & =\frac{-1}{2}\left(\frac{1}{z-1}-\frac{1}{z+1}\right)=\frac{-1}{z^{2}-1} \\
& =-\frac{1}{z^{2}}-\frac{1}{z^{4}}-\frac{1}{z^{6}}-\ldots<0 \text { for } 1<z
\end{aligned}
$$

Indeed by repeated differentiation, we can show that $S(z)$ and all its derivatives are monotonic functions of $z$ for $1<z$ along the real line. When considered in the complex $z$-plane, $S(z)$ in Eq. (39) has singularities at $z=1$ and $z=-1$ and at no other points in the complex plane. Adding any further analytic functions to the expression for $S(z)$ in Eq. (39) would introduce additional singularities in the complex plane. Thus the solution obtained in Eq. (39) is the simplest solution which meets the requirements of the three laws of thermodynamics.

\section{OBTAINING THE PLANCK SPECTRUM}

We have obtained the expression for the entropy $S(z)=S[U /(\omega \hbar / 2)]$ of a harmonic oscillator in terms of the single variable involving oscillator energy divided by its zero-point energy. However, it is usual to give the spectrum for blackbody radiation in terms thermodynamic temperature in practical units $k_{B} T$ and frequency $\omega$ with the spectral density

$$
\rho(\omega, T)=\left(\pi^{2} \omega^{2} / c^{3}\right) U(\omega, T)
$$


where $U(\omega, T)$ is the average energy of a harmonic oscillator (or radiation mode) given in terms of frequency $\omega$ and thermodynamic temperature $T$. We obtain this form by using Eq. (41) rewritten in practical units as

$$
\frac{\omega \hbar / 2}{k_{B} T}=\frac{d\left[S / k_{B}\right]}{d[U /(\omega \hbar / 2)]}=\frac{1}{2} \ln \left(\frac{U /(\omega \hbar / 2)+1}{U /(\omega \hbar / 2)-1}\right) .
$$

Taking the exponential, we find

$$
\exp \left(\frac{\hbar \omega}{k_{B} T}\right)=\left(\frac{U /(\omega \hbar / 2)+1}{U /(\omega \hbar / 2)-1}\right) .
$$

Now solving for $U /(\omega \hbar / 2)$, we obtain

$$
U /(\omega \hbar / 2)=\frac{\exp \left[\hbar \omega /\left(k_{B} T\right)\right]+1}{\exp \left[\hbar \omega /\left(k_{B} T\right)\right]-1}=\operatorname{coth}\left(\frac{\hbar \omega}{2 k_{B} T}\right)
$$

or

$$
U=\frac{1}{2} \hbar \omega \operatorname{coth}\left(\frac{\hbar \omega}{2 k_{B} T}\right)=\frac{\hbar \omega}{\exp \left[\hbar \omega /\left(k_{B} T\right)\right]-1}+\frac{1}{2} \hbar \omega
$$

which is the familiar Planck form, including zero-point energy $U(\omega, 0)=(\hbar / 2) \omega$. Indeed by the use of purely thermodynamic ideas, we are led to the Planck formula including zero-point radiation for blackbody radiation.

\section{REGARDING THE ABSENCE OF ZERO-POINT ENERGY IN THE IN- TERPOLATION FORMULA OBTAINED BY PLANCK}

In classical physics, a dipole harmonic oscillator must come to equilibrium with the surrounding radiation bath where the average energy of the oscillator matches the average energy of the bath radiation modes at the same frequency as the oscillator frequency. Thus the harmonic oscillator treated in the present article must be in equilibrium with a thermal bath which includes both zero-point radiation and thermal radiation above the zero-point radiation as given in Eq. (47).

The unambiguous situation for classical physics is in contrast to the confusion which sometimes occurs in quantum physics. The extrapolation expression based upon experimental data obtained by Planck in 1900 was

$$
U_{P}=\frac{\hbar \omega}{\exp \left[\hbar \omega /\left(k_{B} T\right)\right]-1}
$$


and included no zero-point energy. This result without zero-point energy is often repeated in the textbooks[13] as the energy of the radiation modes of blackbody radiation. Indeed at high temperature, the Planck form (48) goes over to

$$
U_{P}=k_{B} T-\frac{\hbar \omega}{2}+O\left(\frac{\hbar \omega}{k_{B} T}\right)
$$

which still subtracts the zero-point energy compared with the equipartition result $U=$ $k_{B} T$. This situation is in contrast to the analysis in the present article where the oscillator energy at high temperatures was assumed in Eq. (21) to go over to the equipartion result $U=k_{B} T+O\left(\hbar \omega / k_{B} T\right)$. Modern quantum mechanics does include a zero-point energy for a quantum harmonic oscillator.[14] However, the quantum oscillator does not radiate when it is in its energy eigenstates, and so the association with zero-point energy in the radiation field is not obvious.

One must recall that Planck worked from thermodynamics insofar as requiring the Wiendisplacement-theorem form, which depended upon the first two laws of thermodynamics. His blackbody energy expression (48) was obtained as an interpolation (involving experimental data) between the Wien radiation formula, which seemed to fit the high-frequency data well, and the new experimental results of Rubens and Kurlbaum, which suggested a different low-frequency behavior.[15] Once he had obtained his interpolation function in Eq. (48), which provided an excellent fit to the experimental data, he knew that he had to have an entropy expression of the form[15]

$$
S\left(U_{p}, \omega\right)=k_{B}\left[\left(\frac{U_{P}}{\hbar \omega}+1\right) \ln \left(\frac{U_{P}}{\hbar \omega}+1\right)-\left(\frac{U_{P}}{\hbar \omega}\right) \ln \left(\frac{U_{P}}{\hbar \omega}\right)\right]
$$

where $U_{P}$ was the thermal energy of Planck's oscillator in equilibrium with the radiation spectrum measured by the experimentalists. This equation (50) is the same as our Eq. (39) when we introduce the missing constant $k_{B}$ so as to give

$S[U /(\omega \hbar / 2)]=k_{B}\left[\frac{1}{2}\left(\frac{U}{\hbar \omega / 2}+1\right) \ln \left(\frac{U}{\hbar \omega / 2}+1\right)-\frac{1}{2}\left(\frac{U}{\hbar \omega / 2}-1\right) \ln \left(\frac{U}{\hbar \omega / 2}-1\right)-\ln 2\right]$,

and we note the connection between the thermodynamic oscillator energy $U$ and the thermal energy $U_{P}$ used by Planck,

$$
U=U_{P}+\hbar \omega / 2
$$

Planck's energy $U_{P}$ is the thermal energy above the zero-point energy. The Planck form 
in Eq. (48) represents a shift in the oscillator energy over to $U_{P}$ where the entropy $S\left[U_{P} /(\omega \hbar / 2)\right]$ goes to zero as the energy $U_{P}$ goes to zero.

Equations (50) and (51) give the oscillator entropy $S(U, \omega)$ as a function of the energy divided by the frequency. However, we can also obtain the entropy $S(\omega, T)$ as a function of frequency $\omega$ and thermodynamic temperature $T$ by noting from Eq. (45) that $\omega / T$ is a function of $U / \omega$ so that Eq. (44) and the derivative of Eq. (46) can be combined as

$$
\frac{d S(\omega / T)}{d(\omega / T)}=\left(\frac{d S(U / \omega)}{d(U / \omega)}\right)\left[\frac{d(U / \omega)}{d(\omega / T)}\right]=\left(\frac{\hbar \omega}{2 T}\right)\left[-\frac{\hbar}{2 k_{B}} \operatorname{csch}^{2}\left(\frac{\hbar \omega}{2 k_{B} T}\right)\right] .
$$

Then integrating gives

$$
S(\omega / T)=k_{B}\left\{-\ln \left[\sinh \left(\frac{\hbar}{2} \frac{\omega}{k_{B} T}\right)\right]+\frac{\hbar}{2} \frac{\omega}{k_{B} T} \operatorname{coth}\left(\frac{\hbar}{2} \frac{\omega}{k_{B} T}\right)-\ln 2\right\}
$$

where the constant of integration is chosen so that $S \rightarrow 0$ as $T \rightarrow 0$. The zero-point energy makes no contribution to the entropy, and therefore both the Planck form (48) without zeropoint energy and the thermodynamic form (47) including zero-point energy give the same entropy dependence upon frequency and temperature for the harmonic oscillator system.

The discrepancy between the forms in Eqs. (47) and (48) reappears in the calculation of the generalized force associated with the harmonic oscillator system. The generalized force $X$ for the classical simple harmonic oscillator system appears in the fundamental thermodynamic relation $\delta Q=T d S=d U+X d \omega$. From the adiabatic invariant of the mechanical system, we saw that $X=-U / \omega$ where $U$ is the mechanical energy of the mechanical oscillator. In thermodynamics, we expect (from $T d S=d U+X d \omega$ ) that the generalized force is given by

$$
X=-\left(\frac{\partial U}{\partial \omega}\right)_{S} .
$$

Now from equation (51), we see that if $S$ is held constant, then $U / \omega$ must be constant, so $U=\beta \omega$ where $\beta$ is a constant. But then the generalized force follows from $(\partial U / \partial \omega)_{S}=$ $\beta=U / \omega$, so that

$$
X=-\left(\frac{\partial U}{\partial \omega}\right)_{S}=-\frac{U}{\omega}=-\frac{U_{P}+\hbar \omega / 2}{\omega}=-\frac{U_{P}}{\omega}-\frac{\hbar}{2},
$$

involving the same connection $X=-U / \omega$ as was given in Eq. (2). The contribution of the classical zero-point energy is absent in the Planck form (48). Thus the use of the Planck energy $U_{P}$, which excludes the zero-point energy, requires the introduction of an additional 
constant $-\hbar / 2$ at all temperatures in the expression (56) for the generalized force $X$. Even at low temperature $T \approx 0$, where the Planck thermal energy $U_{P}$ approaches zero, there is still a non-zero generalized force given by $X \approx-\hbar / 2$. In classical physics, this force arises from the random radiation associated with zero-point radiation. In quantum theory, this generalized force is a "quantum force" which is different from that associated with thermal energy. As far as classical physics is concerned, the omission of the zero-point energy in the expression (48) is misleading in suggesting that thermodynamic effects vanish as the temperature goes toward absolute zero. The inclusion of an explicit zero-point energy in the system energy, as in Eq. (47), fits with the thermodynamics of classical physics and is far more natural than its absence.

It is also interesting that the second derivative of the entropy with respect to the energy in Eq. (42) can be rewritten in the form associated with Einstein's ideas of energy fluctuations.[16][17] Thus Eq. (42) becomes in practical units

$$
\frac{d^{2} S}{d U^{2}}=-\frac{k_{B}}{U^{2}-U_{0}^{2}}=-\frac{k_{B}}{U^{2}-(\hbar \omega / 2)^{2}}
$$

and associates the entropy $S$ with the energy fluctuations (of the oscillator or radiation mode) above the zero-point energy fluctuations. However, this equation can also be rewritten in terms of the energy $U_{P}$ corresponding to Planck's shift of the energy by the amount of the zero-point energy, $U=U_{P}+U_{0}=U_{P}+\hbar \omega / 2$. In the form using the energy $U_{P}$, the equation becomes

$$
\frac{d^{2} S}{d U^{2}}=-\frac{k_{B}}{\left(U_{P}+U_{0}\right)^{2}-U_{0}^{2}}=-\frac{k_{B}}{U_{P}^{2}+2 U_{0} U_{P}}=-\frac{k_{B}}{U_{P}^{2}+\hbar \omega U_{P}} .
$$

It is this last form involving $U_{P}$ which corresponds to the analysis given by Einstein in 1909. Einstein interpreted the fluctuations as involving a "wave-like" contribution $U_{P}^{2}$ and a "particle-like" (photon) contribution $\hbar \omega U_{P}$. Connecting the energy $U_{P}$ to the number $N$ of photons of frequency $\omega$ as $U_{P}=N \hbar \omega$, the entropy (which follows as in Eq. (50)) goes to zero as the number $N$ of photons of energy $\hbar \omega$ goes to zero.

Planck and Einstein's use of the thermal energy $U_{P}$ without zero-point energy was natural at the turn of the 20th century since the experimental bolometric measurements of thermal radiation involved only the contributions of sources at temperatures above the temperature of the detectors, and thus did not measure zero-point energy. A century later, by Casimirforce[18] measurements, [19] it has become possible (within a classical understanding) to measure all the radiation (including the zero-point radiation) surrounding two parallel con- 
ducting plates. Although the generalized force $X$ given in Eq. (56) for a hypothetical simple harmonic oscillator (associated with the energy dependence on the frequency $\omega$ ) may not be accessible at the atomic level, the generalized force involving random radiation surrounding two conducting parallel plates is indeed measurable.[19] The forces between conducting parallel plates are the radiation-mode analogues of the oscillator generalized force $X$. The Planck form (47) involving photons for the blackbody radiation spectrum, which appears in the modern physics textbooks and in quantum statistical mechanics, 13] does not include zero-point energy and goes to zero as the thermodynamic temperature goes to zero. Thus the photons of the Planck spectrum make no contribution to the Casimir forces between conducting parallel plates at zero temperature. Quantum theory must introduce a separate "quantum mechanical" calculation for the Casimir force at zero temperature, and then adds finite-temperature corrections involving thermal excitations (photons) at non-zero temperature. This is exactly analogous to the need for a "quantum mechanical" generalized force $X$ for an oscillator at zero temperature. Classical theory, which uses the full thermodynamic expression (47) including the zero-point radiation for the energy of a radiation mode, needs only a single calculation to obtain the Casimir force at any temperature. [20] In addition to the need to separate a "quantum behavior" at zero temperature and a thermal contribution (due to photons) at positive temperature, there are also arguments among quantum theorists as to whether or not the Casimir effect at zero temperature should actually be associated with quantum zero-point energy. [23] Within classical physics, there is no ambiguity; Casimir forces are associated with changes in the energy of zero-point radiation. [24]

\section{DISCUSSION}

For over a century now, physicists have presented various theoretical explanations for the Planck spectrum, some based upon quantum theory[21] and some based upon classical theory. [22] However, one may wonder what is the minimal set of assumptions necessary to derive the experimentally observed spectrum. Thermodynamics, which was developed during the 19th century, has provided fundamental ideas of absolute generality. The first two laws of thermodynamics provided the basis for Boltzmann's derivation of the StefanBoltzmann relation $\mathcal{U}=a_{S} T^{4} V$ connecting the thermal energy $\mathcal{U}$ with the temperature $T$ and volume $V$ for the thermal radiation in a closed container at thermal equilibrium. These 
same two laws of thermodynamics, applied to the reversible adiabatic compression of thermal radiation, allowed Wien to derive his displacement theorem in 1893. Planck's enthusiasm for thermodynamics led him to consider the problem of blackbody radiation at the end of the 19th century; however, he became convinced that the information available from the first two laws of thermodynamics had been exhausted by Wien in obtaining the displacement theorem. Therefore he turned to statistical ideas after his successful experimental-databased interpolation giving the Planck spectrum (without zero-point radiation). When the third law of thermodynamics was introduced in the first decade of the 20th century, the law was associated with the developing quantum theory. Apparently physicists never looked back to see whether thermodynamics (now including the third law) might provide the crucial missing function in Wien's displacement theorem.

The thermodynamics of thermal radiation is tightly connected with the thermodynamics of the harmonic oscillator since the behavior of each normal mode of oscillation for radiation takes the simple-harmonic-oscillator form. Indeed, it was Planck who first pointed out that a small classical dipole oscillator would come to thermal equilibrium with an average energy which was the same as the average energy of the random-radiation normal modes at the same frequency as the oscillator. Now the thermodynamics of the harmonic oscillator is virtually never discussed in connection with fundamental thermodynamics rather than in connection with statistical mechanics. Yet the thermodynamics of the simple harmonic oscillator takes a very simple form. Fifteen years ago, it was pointed out[10] that the application of the first two laws of thermodynamics to a harmonic oscillator could lead easily to the information corresponding to Wien's displacement theorem involving the existence of one unknown thermodynamic potential function $\phi(\omega / T)$ depending upon the oscillator frequency $\omega$ divided by the thermodynamic temperature $T$. At that time it was noted that the unknown function $\phi(\omega / T)$ allowed two natural limits which made the oscillator energy $U(\omega, T)$ independent of one of its two variables; the limits corresponded to energy equipartition $U \rightarrow k_{B} T$ at high temperature, and zero-point energy $U \rightarrow \hbar \omega / 2$ at low temperature. It was then suggested that "the smoothest interpolation" between these two limits led to the Planck spectrum. Although the analysis may be compelling physics, the idea of a "smoothest interpolation" is not standard mathematics.

In the present article, we have attempted to go back to the question of the blackbody spectrum within classical physics from the vantage point of all three laws of thermodynamics, 
now including the third law which was not used by Wien or Planck at the turn of the 20th century. We have attempted to use simply the laws of thermodynamics and mathematical function theory to obtain as much information as possible. We find that the third law of thermodynamics forces the idea of zero-point energy for the classical harmonic oscillator. Of course, the idea of a zero-point energy is now associated in the minds of many physicists with quantum theory. However, our work does not require any assumption regarding discrete energy processes. In addition to requiring that an oscillator have a non-zero energy in thermal equilibrium at thermodynamic absolute zero, the laws of thermodynamics provide sufficiently stringent conditions that one can use them alone to derive the Planck blackbody radiation spectrum including zero-point radiation. Finally, we point out that the inclusion of classical zero-point energy, which is automatic from the thermodynamic point of view, involves more natural force ideas than the Planck expression which omits the zero-point energy.

[1] T. S. Kuhn, Black-Body Theory and the Quantum Discontinuity 1894-1912 (Oxford U. Press, New York 1978), p. 6 and p. 346.

[2] A. Hermann The Genesis of Quantum Theory (1899-1913) (translated by C. W. Nash) (MIT Press, Cambridge, MA 1971), pp. 15-17.

[3] See, for example, Kuhn in ref. 1, pp. 213-214, or Hermann in ref. 2, pp. 128-130.

[4] See, for example, F. Reif, Fundamentals of Statistical and Thermal Physics (McGraw-Hill, New York 1985), pp. 119-123 or C. Garrod, Statistical Mechanics and Thermodynamics (Oxford U.P., New York, 1995), p. 124.

[5] T. H. Boyer, "Third Law of Thermodynamics and Electromagnetic Zero-Point Radiation," Phys. Rev. D1, 1526-1530 (1970).

[6] I have yet to find a single textbook which considers the thermodynamics of the harmonic oscillator aside from its appearance in the application of statistical mechanics.

[7] See, for example, H. Goldstein, C. Poole, and J. Safko, Classical Mechanics 3rd ed. (AddisonWesley, New York, 2002), p. 549. For an elementary calculation of the adiabatic invariant for a harmonic oscillator, see, for example, T. H. Boyer, "A Connection Between the Adiabatic Hypothesis of Old Quantum Theory and Classical Electrodynamics with Classical Electro- 
magnetic Zero-Point Radiation," Phys. Rev. A18, 1238-1245 (1978). The most famous and familiar example of this adiabatic invariant involves the slow change in the length of a pendulum.

[8] M. Planck, The Theory of Heat Radiation (Dover, New York 1959). For a brief treatment, see for example, B. H. Lavenda, Statistical Physics: A Probabilistic Approach (Wiley, New York 1991), pp. 73-74.

[9] F. W. Sears and G. L. Salinger, Thermodynamics, Kinetic Theory, and Statistical Mechanics 3rd ed. (Addison-Wesley, Reading, MA 1986), pp. 149-150 and p. 166.

[10] T. H. Boyer, "Thermodynamics of the harmonic oscillator: Wien's displacement law and the Planck spectrum," Am. J. Phys. 71, 866-870 (2003).

[11] Our equation (12) is analogous to Eq. (6-9) on page 150 of Sears and Salinger in ref. 9.

[12] The choice of rational or practical units is discussed, for example, by C. Kittel, Elementary Statistical Mechanics (Wiley, New York 1958), pp. 27, 32-33, or by Garrod, in ref. 4, p. 120.

[13] See for example, R. Eisberg and R. Resnick, Quantum Physics of Atoms, Molecules, Solids, Nuclei, and Particles, 2nd ed ( Wiley, new York 1985), p. 16; or P. M. Morse, Thermal Physics (Benjamin/Cummings, Reading, MA 1981), p. 336; or D. J. Griffiths, Introduction to Quantum Mechanics 2nd ed. (Pearson, Upper Saddle River, NJ 2005), p. 244 and p. 355.

[14] See for example, Griffiths in ref. 13, p. 46.

[15] See, for example, Hermann in ref. 2, pp. 12-14.

[16] A. Einstein, "Zur gegenwärtigen Stand des Strahlungsproblems," Phys. Zeits. 10, 185-193 (1909).

[17] T. H. Boyer, "Classical Statistical Thermodynamics and Electromagnetic Zero-Point Radiation," Phys. Rev. 186, 1304-1318 (1969).

[18] H. B. G. Casimir, "On the attraction between two perfectly conducting plates," Proc. Ned. Akad. Wetenschap. 51, 793-795 (1948).

[19] M. J. Sparnaay, "Measurement of the attractive forces between flat plates," Physica (Amsterdam) 24, 751-764 (1958); S. K. Lamoreaux, "Demonstration of the Casimir force in the 0.6 to $6 \mu \mathrm{m}$ range," Phys. Rev. Lett. 78, 5-8 (1997): 81, 5475-5476 (1998); U. Mohideen, "Precision measurement of the Casimir force from 0.1 to $0.9 \mu \mathrm{m}$," ibid. 81, 4549-4552 (1998); H. B. Chan, V. A. Aksyuk, R. N. Kleinman, D. J. Bishop, and F. Capasso, "Quantum mechanical actuation of microelectromechanical systems by the Casimir force," Science 291, 1941-1944 
(2001): G. Bressi, G. Carugno, R. Onofrio, and G. Ruoso, "Measurement of the Casimir force between parallel metallic surfaces," Phys. Rev. Lett. 88, 041804(4) (2002).

[20] See S. K. Lamoreaux, "Resource letter CF-1: Casimir Force," Am. J. Phys. 67, 850-861 (1999).

[21] See, for example, Griffiths in ref. 13, p. 243-244, or Reif in ref. 4, pp. 373-378.

[22] See, for example, T. H. Boyer, "Blackbody Radiation in Classical Physics: A Historical Perspective," Am. J. Phys. 86, 495-509 (2018).

[23] See for example, R. L. Jaffe, "Casimir effect and the quantum vacuum," Phys. Rev. D 72, 021301(R) (2005).

[24] T. H. Boyer, "Van der Waals Forces and Zero-Point Energy for Dielectric and Permeable Materials," Phys. Rev. A9, 2078-2084 (1974).

(revised September 10, 2018) 